\title{
Papillary Thyroid Microcarcinomas: a 25 Years Retrospective, Institutional Study of 255 Cases
}

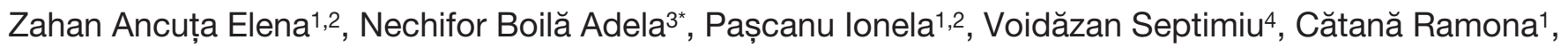 \\ Fișus Andreea ${ }^{3}$, Borda Angela 3,5
}

\author{
Departament of Endocrinology, Emergency County Hospital, Tirgu Mures, Romania \\ 2 Departament of Endocrinology, University of Medicine and Pharmacy of Tirgu Mures, Romania \\ ${ }^{3}$ Departament of Histology, University of Medicine and Pharmacy of Tirgu Mures, Romania \\ 4 Departament of Epidemiology, University of Medicine and Pharmacy of Tirgu Mures, Romania \\ 5 Departament of Pathology, Emergency County Hospital, Tirgu Mures, Romania
}

\begin{abstract}
Background: Papillary thyroid microcarcinoma (PTMC) defines a group of papillary thyroid carcinomas, incidentally discovered, measuring $1 \mathrm{~cm}$ or less. The aim of our study was to evaluate the incidence and the pathological characteristics of PTMCs in our institution in the last 25 years, with special emphasis on the prognostic factors related to PTMCs. Material and methods: We performed a retrospective, cohort study on 255 PTMCs, registered in the Department of Pathology, Tîrgu-Mureș Emergency County Hospital between 1990 and 2014 . Results: A significant increase in the incidence of PTMCs was observed in the 2004-2014 period, compared to 1990-2003 (7.6\%, 229 PTMCs/3005 thyroid specimens vs. 1.2\%, 23 PTMCs/1885 thyroid specimens). Conventional PTMCs accounted for most of PTMC cases ( $n=123$ cases, $48.2 \%)$, followed by the follicular variant of PTC ( $n=117$ cases, $45.9 \%)$. The increasing incidence of PTMCs was associated with a significant increase in the routine number of blocks sampled per case over the study period ( $R 2=0.72, p<0.001)$. By means of the univariate and multivariate analysis, three risk factors were predictive for extrathyroidal extension in PTMCs: multifocality (Odds ratio [OR] 4.97, p-0.002), tumor size $\geq 5 \mathrm{~mm}$ (OR 8.97, p-0.008) and lymph node involvement (OR $17.66 \mathrm{p}<0.005)$. Conclusion: The incidence of PTMCs has revealed a significant increasing trend in our institution over the last 25 years. Multifocality, lymph node involvement and tumor size $\geq 5 \mathrm{~mm}$ were found to be risk factors for extrathyroidal extension. These prognostic factors must be evaluated and clearly mentioned in the pathological report, to help a correct estimation of biological potential of the lesion and an appropriate postoperative management.
\end{abstract}

Keywords: microcarcinoma, incidence, number of blocks, extrathyroidal extension, multifocality

Received: 13 August 2015 / Accepted: 03 September 2015

\section{Introduction}

Papillary thyroid carcinoma (PTC) is the most common thyroid malignancy, with a significant increasing incidence in the last four decades, as reported by many studies around the world [1-4], but with no increase in the mortality rate $[2,4]$. This increase is mainly due to incidentally found PTCs, defined by World Health Organization (WHO) as papillary thyroid microcarcinomas (PTMC) $[1,2,5-7]$. Many factors can be attributed to the increasing incidence of PTMCs. First of all, the increasing number of total thyroidectomies performed for multinodular goiter and a more careful sampling of the thyroid specimens $[2,5,7,8,9]$. Then, the more or less controlled increasing number of thyroid echography. Other imaging examinations performed for different pathologies in the neck region are being highly sensible and may also detect thyroid lesions of less than $1 \mathrm{~cm}[8,10]$. It is thought that in the great majority of cases, the clinical behaviour of these lesions could have been insignificant if left undiscovered [11]. And of course, we should also mention some general factors could be involved in the thyroid carcinogenesis, like: ionizing radiations, lifestyle habits and environmental pollutants $[2,12]$.

* Correspondence to: Adela Nechifor Boilă

E-mail: adelanechifor@gmail.com
It is estimated that PTMC accounted for up to $40 \%$ of all PTCs in the period 2004 to 2008 in the United States [13]. Similar trends have been observed in France and many other countries around the world $[14,15]$. In autopsy series, the prevalence of PTMCs varies from 3 to $36 \%$ and is largely dependent on the thyroid sampling technique and from country to country [16-18]. In surgical thyroidectomies performed for benign lesion, the prevalence of PTMCs varies from $5 \%$ to $17 \%[19,20]$.

PTMCs have an indolent course and an excellent prognosis and therefore are usually considered of little clinical significance $[11,21]$. However, rarely PTMCs may have a more aggressive clinical behaviour $[8,15,22]$. Some clini$\mathrm{cal}$, pathological and, more recently, molecular prognostic factors have been identified to foresee the tumour progression and prognosis of patients with PTMC $[15,23,24]$. The most important clinical prognostic factors include the patient's age 45 years old and older at the time of diagnosis, the male gender [24,25] and high postoperative levels of stimulated thyroglobulin [26]. The pathological indicators of an unfavourable prognosis are the extrathyroidal extension, lymph node involvement and distant metastasis, so called "aggressive" variants of PTC [23,27-30].

Some molecular biomarkers have been associated with PTMCs aggressiveness and clinical behaviour but their value is still under debate $[31,32]$. 
In this study we aim to evaluate the incidence and the pathological characteristics of PTMCs in our institution in the last 25 years, with special emphasis on the prognostic factors related to PTMCs.

\section{Materials and methods Database and cases definition}

We performed a 25 years, retrospective, cohort study on 4890 cases of thyroid pathology registered in the Department of Pathology, Tîrgu - Mureș Emergency County Hospital between January 1990 and December 2014.

Clinicopathological data on the study cases were retrieved from database registers and pathological reports. The following variables were included when analysing the incidence and prognostic factors of PTMC cases: age at diagnosis, gender, surgical procedure, tumor histological type, size, multifocality (unilateral or bilateral), extrathyroidal extension, lymph node involvement and surgical resection margins. The type of the surgical procedure was also recorded: lobectomy with or without isthmectomy, subtotal thyroidectomy, total thyroidectomy and total thyroidectomy with central or lateral neck compartment dissection.

PTMCs were referred to PTCs incidentally found, that measured $1 \mathrm{~cm}$ or less. The diagnosis of PTC was exclusively based on nuclear features: enlargement, overlapping, irregularity of the nuclear contours, grooves, clearing or a ground glass appearance and nuclear pseudoinclusions. Conventional PTCs had a characteristic papillary architecture that was pure or admixed with a variable proportion of follicles. Tumors defined as follicular variant of PTC were composed of small to medium sized, irregularly shaped follicles, with characteristic PTC nuclear changes in most of the cells lining these follicles and virtually no papillary structures. The diagnosis of other rare variants of PTC (tall cell, oncocytic, Warthin-like) was made in accordance to the WHO criteria [1].

A standardized sampling technique was applied to all cases in our study consisting of one tissue samples from the upper third, middle part and lower third of each thyroid lobe, respectively (in general 3 to 6 tissue samples per case) $[33,34]$. When necessary, for accurate diagnosis of the thyroid lesion, the number of samples was multiplied.

The 2009 TNM staging system (tumor size, extrathyroidal extension, lymph node metastasis, distant metastasis) was applied for all the cases included in the study [35].

The following prognostic factors have been evaluated: the age: 45 years old and older, the gender, tumor size $\geq 5$ $\mathrm{mm}$, histological variant of PTC, extrathyroidal extension, multifocality, lymph node involvement.

Age at diagnosis is the most important clinical factor in the UICC/AJCC-Union International Centre le Cancer/ American Joint Cancer Committee-staging system for thyroid cancer. The patients younger than 45 years belong to stage I, regardless of the primary tumor TNM based staging system, while the patients from 45 years-old and older belong in the stage I-IV, depending on the TNM - based staging system [35].

Extrathyroidal extension was defined as tumor penetration through the thyroid capsule into the adjacent tissues, with invasion into immediate perithyroidal soft tissues or sternothyroid muscle (TNM stage T3 tumors) [35].

Multifocality was defined as the presence of two or more isolated/non-contiguous tumor foci in the resected thyroid gland [2].

The lymph node involvement was considered positive if at least one positive lymph node was present.

In order to analyze the incidence, clinical and pathological features of PTMCs, we subdivided the total series in two main groups, according to the time of diagnosis: group one (period 1990-2003) and group two (after 2004). The cut-off (the year 2004) has been chosen in accordance to the introduction of the new WHO classification of Endocrine Tumors [1].

\section{Data analysis}

Statistical analysis was performed using the Statistical Package for Social Sciences (SPSS, version 20, Chicago, IL, USA). Data were labelled as nominal or quantitative variables. Nominal variables were characterized by means of frequencies. Quantitative variables were tested for normality of distribution using Kolmogorov-Smirnov test and were described by mean \pm standard deviation or median and percentiles (25\%; 75\%), whenever appropriate. The frequencies of nominal variables were compared with a chi-square test. Differences in the mean or median between groups were analyzed using the $t$ test and ANOVA test. The odds ratio (OR) and the $95 \%$ confidence interval (CI) for relationships between variable dependent and each variable independent were calculated using binary logistic regression (univariate and multivariate logistic regression). The level of statistical significance was set at $\mathrm{p}<0.05$.

\section{Results}

\section{Incidence, clinical and pathological characteristics of PTMC cases}

From a total of 4890 tumoral and non-tumoral thyroid lesions, registered in our department over the last 25 years, 255 (5.2\%) were PTMCs. Complete data including data from pathological reports were available for 237 (93\%) PTMCs.

The clinicopathological characteristics of PTMC cases are summarized in Table I. The majority of cases occurred in women ( $n=215$ cases, $90.5 \%$ ), with the female to male ratio was 9.7:1. The mean age at diagnosis was $51.5 \pm$ 11.89 years-old, ranging from 14 to 77 years-old.

Regarding the surgical procedure, total thyroidectomy, subtotal thyroidectomy, lobectomy and total thyroidectomy with central or lateral neck compartment dissection were performed in 157 (70.7\%), 30 (13.5\%), 27 (12.2\%) and $8(3.6 \%)$ cases, respectively. 
Table I. Clinicopathological characteristics of papillary thyroid microcarcinoma cases in our institution.

\begin{tabular}{|c|c|c|c|}
\hline Characteristics & PTMC (\%) & $\begin{array}{c}\text { Tumor }<5 \mathrm{~mm} \\
(\%)\end{array}$ & $\begin{array}{c}\text { Tumor } \geq 5 \mathrm{~mm} \\
(\%)\end{array}$ \\
\hline Total & 255 & & \\
\hline $\mathrm{a}$ & 237 & $115(48.5)$ & $122(51.5)$ \\
\hline Mean age (years-old) & $51.54 \pm 11.89$ & $51.27 \pm 1.147$ & $51.78 \pm 1.114$ \\
\hline $\begin{array}{c}\mathrm{p} \\
\text { Age (years) }\end{array}$ & \multicolumn{2}{|c|}{ Age (years) } & 0.7499 \\
\hline$<45$ & $66(27.8)$ & $35(30.4)$ & $31(27.4)$ \\
\hline$\geq 45$ & $171(72.2)$ & $80(69.6)$ & $91(72.6)$ \\
\hline $\mathrm{p}$ & & & 0.388 \\
\hline $\begin{array}{l}\text { Female } \\
\text { Male }\end{array}$ & $\begin{array}{c}215(90.7) \\
22(9.3)\end{array}$ & $\begin{array}{c}101(87.8) \\
14(12.2)\end{array}$ & $\begin{array}{c}114(93.4) \\
8(6.6)\end{array}$ \\
\hline $\mathrm{pb}$ & & & 0.136 \\
\hline $\begin{array}{l}\text { Tumor size } \\
\text { (mean, mm) }\end{array}$ & $5.423 \pm 4.685$ & & \\
\hline \multicolumn{4}{|l|}{ pTstagec } \\
\hline pT1a & 217 (91.6) & $113(98.3)$ & $104(85.2)$ \\
\hline рT3 & $20(8.4)$ & $2(1.7)$ & $18(14.8)$ \\
\hline $\mathrm{p}$ & & & 0.0001 \\
\hline $\begin{array}{l}\text { Lymph node } \\
\text { involvement }\end{array}$ & $4(1.8)$ & $2(1.7)$ & $2(1.6)$ \\
\hline$p$ & & & 0.952 \\
\hline Multifocality & $54(22.8)$ & $20(17.4)$ & $34(27.9)$ \\
\hline $\mathrm{pb}$ & & & 0.040 \\
\hline $\begin{array}{l}\text { Extrathyroidal } \\
\text { extension }\end{array}$ & $19(8)$ & $2(1.7)$ & $17(13.9)$ \\
\hline $\mathrm{pb}$ & & & $<0.0001$ \\
\hline
\end{tabular}

Surgical resection margin

\begin{tabular}{cccc} 
positive & $4(1.7)$ & 0 & $4(3.3)$ \\
$\mathrm{pb}$ & & & 0.040 \\
\hline
\end{tabular}

PTMC - Papillary thyroid microcarcinoma

CPTMC - conventional PTMC

FVPTMC - follicular variant of PTMC

a - number of cases with complete available data (database registries and pathological reports)

$\mathrm{b}$ - The $\mathrm{p}$ value was the result of comparison between PTMC with tumor size $<5 \mathrm{~mm}$ and

PTMC with tumor size $\geq 5 \mathrm{~mm}(\mathrm{p}<0.05)$

c - TNM Classification of malignant tumors.7th Edition ed. Springer (2009).

PTMCs represented $35.1 \%$ of all 727 PTC cases diagnosed in our department over the study period, with a significant increasing trend of this ratio in the last ten years, compared to the previous period (2004-2014: 229 PTMCs/ 583 PTC, 39.3\% vs. 1990-2003: 23 PTMCs/ 144 PTC, $15.9 \%, \mathrm{p}<0.0001)$. Conventional PTMCs accounted for most of the PTMC cases ( $n=123$ cases, $48.2 \%$ ), followed by the follicular variant of PTMCs $(\mathrm{n}=117$ cases, $45.9 \%)$. Rare variants of PTMC included: oncocytic ( $n=7$ cases), Whartin-like ( $n=7$ cases) and tall-cell $(n=1$ cases) variants.

A significant increase in the incidence of PTMCs was observed in the period 2004-2014 (7.6\%, 229 PTMCs/3005 thyroid specimens), compared to the period 1990-2003 (1.2\%, 23 PTMCs/ 1885 thyroid specimens $\mathrm{p}<0.001)$, but the total number of thyroid procedures also increased significantly in this period (1885 thyroid specimens 19902003 vs. 3005 thyroid specimen in 2004-2014, $\mathrm{p}<0.001$ ) (Figure 1).

The mean number of paraffin blocks has also significantlly increased over the study period $\left(R^{2}=0.73, p<0.001\right)$, parallel to the increasing incidence of PTMCs. Figure 2 further demonstrates the highly significant association between the mean number of blocks taken each year and the number of reported PTMCs $\left(\mathrm{R}^{2}=0.72, \mathrm{p}<0.0001\right)$. A significant increase in the number of bocks sampled per case was noticed in 2004-2014 (6.8 blocks per case), compared to $1990-2003$ (3.8 blocks per case) $(\mathrm{p}<0.001)$.

\section{Prognostic factors of PTMC cases}

\section{Age and gender}

Univariate and multivariate logistic regression analysis showed that patient's age over 45 years-old and male gender were not associated with more aggressive behaviour of PTMC.

\section{Tumor size}

More than half of the tumors ( $n=122$ cases, $51.5 \%$ ) had $5 \mathrm{~mm}$ or more in size, and these tumors were found to be associated with a higher TNM tumor stage $(p<0.0001)$, a higher risk for multifocality $(p=0.04)$, extrathyroidal extension $(p<0.0001)$ and positive surgical resection margins $(\mathrm{p}=0.04)$, compared to smaller tumors (less than $5 \mathrm{~mm}$ in size) (Table I).

\section{Histologic variants of PTMC}

Conventional PTMCs revealed a higher TNM tumor stage ( $14.3 \%$ vs. $1.7 \%$ p $<0.0001)$ and a higher rate of extrathy-

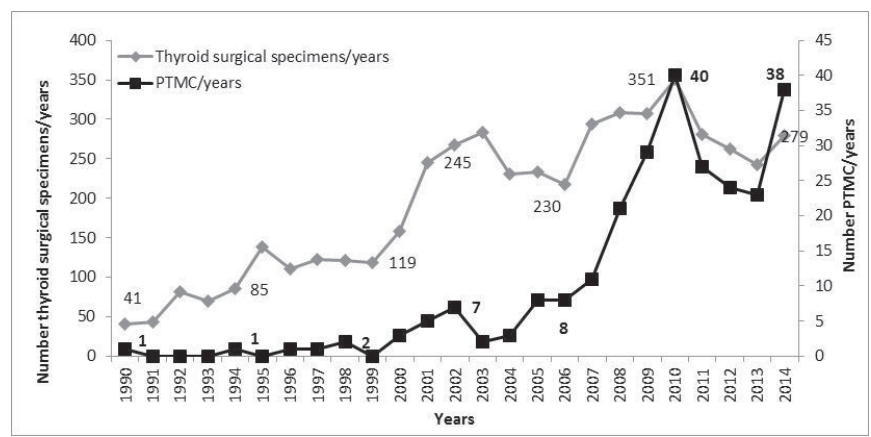

Fig. 1. The distribution of the papillary thyroid microcarcinomas and thyroid surgical specimen in different periods of the study

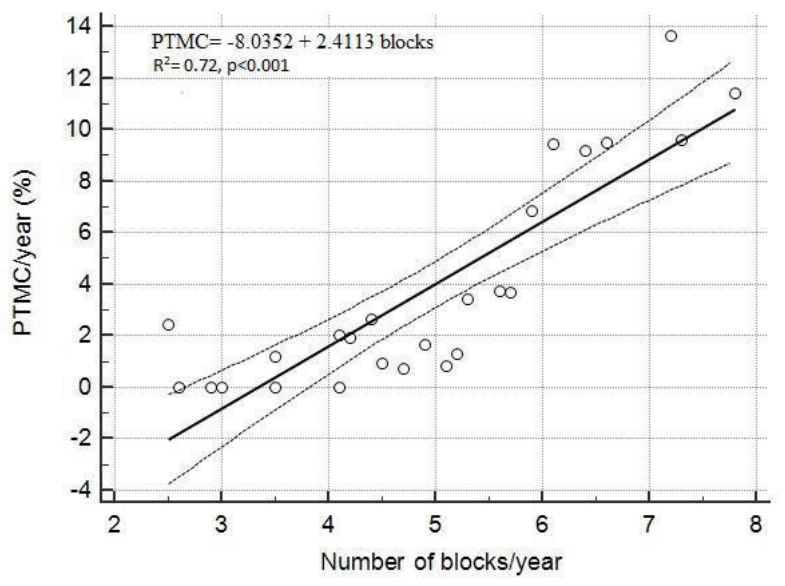

Figure 2. Regression line with $95 \% \mathrm{Cl}$ showing the association between the number of papillary microcarcinomas (as a percentage of the total number of cases diagnosed every year) and the average number of blocks taken per year. 
roidal extension, compared to the follicular variant of PTMCs $(14.3 \%$ vs. $1.7 \%, \mathrm{p}<0.0001)$. Follicular variant of PTMC cases were more prevalent in men, compared to conventional PTMCs, which were much more prevalent in women $(14.5 \%$ vs $3.8 \%$., $\mathrm{p}=0.006)$. No statistically significant differences were observed between conventional PTMCs and Wartin-like, oncocytic or tall cell variants of PTC cases, respectively, with regard to all morphological prognostic indicators.

\section{Extrathyroidal extension}

Nineteen (8\%) PTMCs had extrathyroidal extension. By means of the univariate and multivariate analysis, three risk factors were predictive for extrathyroidal extension in PTMCs: multifocality (Odds ratio [OR] 4.97, p-0.002), tumor size $\geq 5 \mathrm{~mm}$ (OR 8.97, p-0.008), lymph node involvement (OR 17.66 p-0.0056) (Table II).

\section{Multifocality}

The incidence rate of multifocality in PTMCs was $22.8 \%$ (54 cases). Our results showed that multifocal PTMCs were significantly more frequently in males (OR 2.86, $\mathrm{p}-0.03)$ and extrathyroidal extension (OR 4.95; p-0.002) (Table III).

\section{Lymph node involvement}

The prevalence of lymph node involvement in PTMC cases was $1.8 \%$ and this parameter was not significantly associated with the age 45 years old and older, the multifocality rate and the extrathyroidal extension.

\section{Discussions}

Our study revealed an important increase in the incidence of PTMC in our institution over the last 25 years (19902014). The incidence of PTMC was characterized by a statistically significant increasing trend after 2004 compared to the previous period $(7.6 \%, 229$ PTMCs/3005 thyroid specimen $v$ s. $1.2 \%, 23$ PTMCs/ 1885 thyroid specimen). Similar results have been reported in many countries around the world, and especially in Europe and North America $[4-7,13,14]$.

Many factors can be attributed to the increasing incidence of PTMCs. The increasing number of thyroid echography, more or less controlled, the increasing number of total thyroidectomies performed for multinodular goiter and a more careful sampling of the thyroid specimens are among the most important factors to be considered [2,5,7-9]. Population-level data supports this hypothesis, by correlating the increasing thyroid cancer incidence to the increasing use of thyroid ultrasound and fine needle aspiration (FNA), that were introduced in the early 1980 s for thyroid cancer diagnosis $[2,4,36]$. Udelsman et al. have showed that $57 \%$ and $49 \%$ of the variability in the USA state-level incidence of thyroid cancer for males and females, respectively, can be explained by the regional variation in the density of endocrinologists, general surgeons and employment of cervical ultrasonography [10].

The sampling method of the thyroid was found to be correlated to the increasing incidence of PTMC. Usually, a standardized sampling method is used, consisting in including 3 blocks per thyroid lobe. In some instances this number could be much greater, imposed by the necessity of an accurate diagnosis of specific thyroid lesion. For nonneoplastic multinodular goiters, up to five nodules per thyroid lobe could be sampled, depending on pathologist's experience $[33,34]$. For encapsulated solitary nodules, to detect capsular or vascular invasion multiple samples per nodule are required. Moreover young pathologists, to be sure not to skip a malignant lesion are performing an overmuch sampling method, increasing the number of blocks per thyroid lobe.

Our results indicate that the increasing incidence of PTMCs was significantly correlated to the number sampled blocks. The increasing incidence of PTMCs had followed a parallel trend to the increasing number of blocks sampled per case over the years, and these results were statistically significant (2004 - 2014 - 6.8 blocks per case vs. 1990-

Table II. Univariate and multivariate logistic regression for extrathyroidal extension in papillary thyroid microcarcinoma cases.

\begin{tabular}{|c|c|c|c|c|c|c|}
\hline \multirow{2}{*}{ Independent variable } & \multicolumn{3}{|c|}{ Univariate } & \multicolumn{3}{|c|}{ Multivariate } \\
\hline & OR & {$[95 \% \mathrm{Cl}]$} & $p$ & OR & {$[95 \% \mathrm{Cl}]$} & $\mathrm{p}$ \\
\hline Age ( $\geq 45$ years ) & 0.03 & {$[0.019-0.06]$} & 0.001 & 1.23 & {$[0.33-4.52]$} & 0.76 \\
\hline Gender (male) & 0.85 & {$[0.45-1.62]$} & 0.62 & 0.002 & {$[0.012-0.09]$} & 0.99 \\
\hline Multifocality & 2.95 & {$[1.69-5.16]$} & 0.001 & 4.97 & [1.77 - 13.99] & 0.002 \\
\hline Lymph node metastases & 5.08 & {$[1.7-15.16]$} & 0.002 & 17.67 & [1.35 - 231.04] & 0.005 \\
\hline Tumor size $\geq 5 \mathrm{~mm}$ & 8.2 & {$[4.81-14.01]$} & 0.001 & 8.97 & {$[1.75-46.06]$} & 0.008 \\
\hline
\end{tabular}

OR- Odds ratio, $\mathrm{Cl}$ - 95\% confidence interval, $\mathrm{p}<0.05$, Dependent variable- extrathyroidal extension

Table III. Univariate and multivariate logistic regression for multifocality rate in papillary thyroid microcarcinoma cases.

\begin{tabular}{|c|c|c|c|c|c|c|}
\hline \multirow{2}{*}{ Independent variable } & \multicolumn{3}{|c|}{ Univariate } & \multicolumn{3}{|c|}{ Multivariate } \\
\hline & OR & {$[95 \% \mathrm{Cl}]$} & $\mathrm{p}$ & OR & {$[95 \% \mathrm{Cl}]$} & $\mathrm{p}$ \\
\hline Age ( $\geq 45$ years ) & 0,12 & {$[0,075$ to 0,17$]$} & 0.07 & 1.32 & [0.63-2.80] & 0.46 \\
\hline Gender (male) & 2.88 & [1.69 to 4.92 ] & 0.001 & 2.86 & {$[1.09-7.45]$} & 0.03 \\
\hline Extrathyroidal extension & 3.39 & {$[1.93-5.91]$} & 0.001 & 4.97 & {$[1.77-13.88]$} & 0.002 \\
\hline Lymph node metastases & 1.32 & {$[0.62-14.16]$} & 0.001 & 2.44 & {$[0.27-22.04]$} & 0.42 \\
\hline Tumor size $\geq 5 \mathrm{~mm}$ & 0.28 & {$[0.18-0.41]$} & 0.08 & 1.58 & {$[0.80-3.08]$} & 0.18 \\
\hline
\end{tabular}

OR- Odds ratio, $\mathrm{Cl}$ - 95\% confidence interval, $\mathrm{p}<0.05$, Dependent variable - multifocality 
2003- 3.8 blocks per case, $\mathrm{p}<0.001)$. Similar data were also reported by Grodski et al [9].

Other studies reported similar results. A meta-analysis study performed by Roti et al. has shown that the prevalence of PTMCs was correlated to the sampling method: PTMC was detected in $9.9 \%$ of cases if only one suspected macroscopic lesion was examined and on the contrary, PTMC was found in $15.5 \%$ cases when the entire gland was examined [15].

The female/male ratio in PTMC cases was 9.7/1, a much higher value compared to the $4.2 / 1$ ratio reported by Roti E et al [15] or Besic N et al [24]. This can be related to their higher prevalence of thyroid disease in women compared with men and, therefore, greater access to diagnostic procedures resulting in increased identification of PTMC.

The age at diagnosis is a well-known prognostic factor in patients with PTMC and represents one of the major determinants used in AJCC/UICC staging system (7th Edition ) [35]. In our study, $72.2 \%$ of patients with PTMC were older than 45 years-old at the time of diagnosis, but the extrathyroidal extension, the multifocality, the lymph node involvement and the tumor size more than $5 \mathrm{~mm}$ revealed a similar prevalence compared to younger subjects. In the study of Yu et al [25] they report that the age over 45 years-old and male gender were identified as significant risk factors for overall survival.

Several authors have reported that PTMCs that have $>5$ $\mathrm{mm}$ in size had a higher incidence of lymph node involvement, capsule invasion and multifocality, compared with those $\leq 5 \mathrm{~mm}[29,36]$, whereas other studies have shown no differences [37]. In our study we demonstrated that tumor size (< or $\geq 5 \mathrm{~mm}$ ) was an independent risk factor for extrathyroidal extension. In addition, we found no significant differences in regard to the gender, multifocality, positive surgical resection margins and TNM stage between tumors less than $5 \mathrm{~mm}$ in size, compared to larger tumors ( $\geq 5 \mathrm{~mm}$ ).

Another pathological prognostic factor is the presence of extrathyroidal extension in PTMC, an important risk factor used in TNM- based staging system [35]. In our study, only $8 \%$ of the PTMCs had extrathyroidal extension. Similar data were reported by Malandrino et al. [23] where $7.5 \%$ patients had extrathyroidal extension out of total 1777 PTMC cases. Our results show that the presence of multifocality, lymph node involvement and tumor size $\geq 5 \mathrm{~mm}$ increased the risk for developing a extrathyroidal extension by 4.97, 17.66 and 8.96 folds, respectively. Moon $\mathrm{HJ}$ et al. showed that extrathyroidal extension was statistically significantly associated with tumor size (OR 1.18), infiltrative margins on ultrasound (OR 3.8) and central lymph node involvement at diagnosis time (OR 2.1). However, recurrence was not significantly different in patients with or without extrathyroidal extension [27]. In a recent study Malandrino et al. identified 2 independent prognostic factors for recurrence: the presence of node metastases at diagnosis and extrathyroidal extension [23].
The prevalence of multifocality in PTMC ranges from 18 to $87 \%$, and similar rates have been reported in PTC, as well $[23,30,37]$. In our study, the multifocality rate was $22.8 \%$. The prognostic importance and clinical significance of multifocality in PTMC remains controversial. Some studies demonstrated that patients with multifocal PTMC have an increased risk of regional lymph node involvement and is associated with higher risk of recurrence $[8,30,37]$. Other studies, on the other hand, have reported different results. Neuhold et al., and Ross et al., for example, have shown that multifocality was not an independent risk factor for PTMC recurrence or lymph node metastasis $[21,38]$. More recently, Kim et al. concluded that multifocal PTMC was significantly associated with tumor size, extrathyroidal extension, lymph node metastasis and advanced TNM stage, but did not have significantly different rates of disease persistence or tumor recurrence [39]. In our study, the presence of extrathyroidal extension and the male gender increased the risk for developing a multifocal tumor by 4.95 and 2.86 folds, respectively.

The presence of lymph node involvement increases the risk of disease recurrence $[8,22,23,28,29,37]$. The incidence of lymph node metastasis in PTMC cases varies considerably, ranging from $32 \%$ to $64.1 \%$ or $9.7 \%$ to $42.4 \%$ for central or lateral cervical lymph nodes, respectively $[23,28]$. In our study, lymph node involvement was present in only $1.8 \%$ of total PTMC cases. One explanation could be the low rate of the prophylactic central neck dissection, performed by the surgeons in our institution.

Regarding the histological variant, many studies have shown that follicular variant of PTMC is associated with a more favourable prognosis, compared to conventional PTCs $[8,21,40]$. Indeed, in our study, the conventional PTMCs revealed a higher rate of extrathyroidal extension and more prevalent TNM T3 tumor stage, compared to the follicular variant of PTMC.

An important limitation of our study is the lack of data regarding the mortality and/or disease-specific survival rates in patients with PTMC. However, we succeeded to determine the some risk factors for the presence of extrathyroidal extension, multifocality and lymph node metastases at diagnosis, in our population.

\section{Conclusions}

In summary, the incidence of PTMCs has revealed a significant increasing trend in our institution over the last 25 years. In our study the increasing incidence of PTMC was correlated to the increased number of sampled blocks. Multifocality, lymph node involvement and tumor size $\geq$ $5 \mathrm{~mm}$ were found to be risk factors for extrathyroidal extension. The presence of extrathyroidal extension and the male gender increased the risk of developing multifocality. Therefore, these prognostic factors must be evaluated and clearly mentioned in the pathological report, to help a correct estimation of biological potential of the lesion and an appropriate postoperative management. 


\section{Conflicts in interest}

The authors declare that they have no conflicts of interest.

\section{Acknowledgements}

This paper is supported by the Sectoral Operational Programme Human Resources Development (SOP HRD), financed from the European Social Fund and by the Romanian Government under the contract number POSDRU/159/1.5/S/137390/.

\section{References}

1. DeLellis RA, Lloyd RV, Heitz PU, Eng C. World Health Organization Classification of Tumours. Pathology and genetics of tumours of endocrine organs. Lyon: IARC Press. 2004;p.54-5.

2. YE. Nikiforov, Paul W. Biddinger, Lester D.R. Thompson. Diagnostic Pathology and Molecular Genetics of the Thyroid Second EditionChapter 7 - Thyroid Tumors: Classification, Staging and General Considerations, Chapter 11- Papillary Carcinoma, Lippincott Williams \& Wilkins. 2012;108-118,183-243.

3. Kahn C, Simonella L, Sywak M, et al. Postsurgical pathology reporting of thyroid cancer in New South Wales, Australia. Thyroi. 2012;(22):604-10.

4. Davies L, Welch HG. Current Thyroid Cancer Trends in the United States. JAMA Otolaryngol Head Neck Surg. 2014;140(4):317-322.

5. Kent WD, Hall SF, Isotalo PA, et al. Increased incidence of differentiated thyroid carcinoma and detection of subclinical disease. CMAJ. 2007; (177):1357-61.

6. Hay ID, Hutchinson ME, Gonzalez-Losada T, et al. Papillary thyroid microcarcinoma: a study of 900 cases observed in a 60-year period. Surgery. 2008;(144):980-8.

7. Hughes DT, Haymart MR, Miller BS, et al. The most commonly occurring papillary thyroid cancer in the United States is now a microcarcinoma in a patient older than 45 years. Thyroid. 2011;(21):231-236.

8. Furio Pacini. Thyroid microcarcinoma. Best Practice \& Research Clinical Endocrinology \& Metabolism. 2012;421-429.

9. Grodski S, Brown T, Sidhu S, et al. Increased incidence of thyroid cancer is due to increased pathologic detection. Surgery. 2008;(144):1038-43.

10. Udelsman $R$, Zhang $Y$. The epidemic of thyroid cancer in the United States: The role of endocrinologists and ultrasounds. Thyroid. 2014;24(3):472479.

11. Furio Pacini . Management of Papillary Thyroid Microcarcinoma: Primum Non Nocere! J Clin Endocrinol Metab. 2013;98(4):1391-1393.

12. Kitahara CM, Platz EA, Beane Freeman LE, et al. Physical activity, diabetes, and thyroid cancer risk: A pooled analysis of five prospective studies. Cancer Causes \& Control. 2012;23(3):463-71.

13. Howlander N, Noone AM, Krapcho M, et al. J. SEER Cancer Statistics Review, 1975-2008. National Cancer Institute; Bethesda, MD: http:// seer.cancer.gov/csr/1975_2008/, based on November 2010 SEER data submission, posted to the SEER web site, 2011.

14. Leenhardt L, Grosclaude P, Cherie-Challine L. Increased incidence of thyroid carcinoma in France: a true epidemic or thyroid nodule management effects? Report from the French Thyroid Cancer Committee. Thyroid. 2004;(14):1056-1060.

15. Roti E, degli Uberti EC, Bondanelli $M$, et al. Thyroid papillary microcarcinoma: a descriptive and meta-analysis study. Eur J Endocrinol. 2008;(159):659-673.

16. Martinez-Tello FJ, Martinez-Cabruja R, Fernandez-Martin J, et al. Occult carcinoma of the thyroid. A systematic autopsy study from Spain of two series performed with two different methods. Cancer. 1993;(71):40224029.

17. Harach HR, Franssila KO, Wasenius VM. Occult papillary carcinoma of the thyroid. A "normal" finding in Finland. A systematic autopsy study. Cancer 1985;(56):531-538.

18. Kovacs GL, Gonda G, Vadasz G, et al. Epidemiology of thyroid microcarcinoma found in autopsy series conducted in areas of different iodine intake. Thyroid. 2005;(15):152-7.

19. Sakorafas GH, Stafyla V, Kolettis T, et al. Microscopic papillary thyroid cancer as an incidental finding in patient treated surgically for presumably benign thyroid disease. Journal of Postgraduate Medicine. 2007;(53):2326.

20. De Matos PS, Ferreira AP, Ward LS. Prevalence of papillary microcarcinoma of the thyroid in Brazilian autopsy and surgical series. Endocrine Pathology. 2006;(17):165-173.

21. Neuhold N, Schultheis A, Hermann M. Incidental papillary microcarcinoma of the thyroid-further evidence of a very low malignant potential: a retrospective clinicopathological study with up to 30 years of follow-up. Ann Surg Oncol. 2011;(18):3430.

22. Piana S, Ragazzi M, Tallini G, et al. Papillary thyroid microcarcinoma with fatal outcome: evidence of tumor progression in lymph node metastases: report of 3 cases, with morphological and molecular analysis. Hum Pathol. 2013;44(4):556-565.

23. Malandrino P, Pellegriti G, Attard M, et al. Papillary thyroid microcarcinomas:a comparative study of the characteristics and risk factors at presentation in two cancer registries. JClin Endocrinol Metab. 2013;(98):1427-1434.

24. Besic N, Pilko G, Petric R, et al. Papillary thyroid microcarcinoma: prognostic factors and treatment. J Surg Oncol. 2008;(97):221-5.

25. Yu XM, Wan Y, Sippel RS, et al. Should all papillary thyroid microcarcinomas be aggressively treated? An analysis of 18,445 cases. Ann Surg. 2011;(254):653-660.

26. Robenshtok E, Grewal RK, Fish S, et al. A low postoperative nonstimulated serum thyroglobulin level does not exclude the presence of radioactive iodine avid metastatic foci in intermediate-risk differentiated thyroid cancer patients. Thyroid. 2013;23(4):436-442.

27. Moon HJ, Kim EK, Chung WY. Minimal extrathyroidal extension in patients with papillary thyroid microcarcinoma: is it a real prognostic factor? Ann Surg Oncol. 2011;(18):1916-23.

28. Zhang L, Wei WJ, Ji QH, et al. Risk factors for neck nodal metastasis in papillary thyroid microcarcinoma: a study of 1066 patients. J Clin Endocrinol Metab. 2012;(97):1250-1257.

29. Kim BY, Jung $\mathrm{CH}$, Kim JW, et al. Impact of clinicopathologic factors on subclinical central lymph node metastasis in papillary thyroid microcarcinoma. Yonsei Med J. 2012;(53):924-930.

30. Zhao Q, Ming J, Liu C, et al. Multifocality and total tumor diameter predict central neck lymph node metastases in papillary thyroid microcarcinoma. Ann Surg Oncol. 2013;(20):746-52.

31. Elisei R, Viola D, Torregrossa L,et al. The BRAF (V600E) mutation is an independent, poor prognostic factor for the outcome of patients with lowrisk intrathyroid papillary thyroid carcinoma: single institution results from a large cohort study. J Clin Endocrinol Metab. 2012;(97):4390-4398.

32. Virk RK, Van Dyke AL, Finkelstein A, et al. BRAFV600E mutation in papillary thyroid microcarcinoma: a genotype-phenotype correlation. Mod Pathol. 2013;26(1):62-70.

33. Berger N, Borda A. Pathologie thyroidienne, parathyroidienne et surrenalienne- Techniques d'etude des lesions thyroidienne. Editura Sauramps Medical Montpellier. 2010;(366):29-33.

34. Nikiforov YE, Biddinger PW, Thompson LDR. Diagnostic Pathology and Molecular Genetics of the Thyroid Second Edition - Chapter 19 - Gross Examination, Lippincott Williams \& Wilkins. 2012;403-409.

35. Sobin LH, Gospodarowicz MK, Wittekind C. UICC TNM Classification of Malignant Tumours. 7th ed. Wiley-Liss; New York NY: 2009.

36. Friguglietti CU, Dutenhefner SE, Brandao LG, et al. Classification of papillar thyroid microcarcinoma according to the size and fine-needle aspiration cytology: behavior and therapeutic implications. Head Neck. 2011;(33):696-701.

37. Buffet C, Golmard JL, Hoang C, et al. Scoring system for predicting recurrences in patients with papillary thyroid microcarcinoma. Eur $J$ Endocrinol. 2012;(167):267-275.

38. Ross DS, Litofsky D, Ain KB, et al. Recurrence after treatment of micropapillary thyroid cancer. Thyroid. 2009;(19):1043-8.

39. Kim K, Kim S, Lee YS, et al. Prognostic significance of tumor multifocality in papillary thyroid carcinoma and its relationship with primary tumor size: A retrospective study of 2,309 consecutive patients. Annals of Surgical Oncology. 2015;22(1):125-31.

40. Ortiz Sebastian S, Rodriguez Gonazles JM, Parilla Paricio P, et al. Papillary thyroid carcinoma: prognostic index for survival including the histological variety. Arch Surg. 2000;(135):272-277. 